\title{
Notes on Some Oscillation Criteria
}

\section{Hiroshi ONOSE*}

This note is concerned with the property of solutions of the ordinary differential equation. The first section is devoted to some oscillation criteria and the second one is devoted to the periodicity.

1. This section is concerned with the behavior near $t=\infty$ of solutions of the differential equations of the form

(*)

$$
x(t)^{(n)}+f(t)_{\xi}\left(x, x^{\prime}, \ldots, x^{(n-1)}\right)=0(n>1) .
$$

A solution is said to oscillate near $t=\infty$ if it has no largest zero and it becomes an important problem how to distinguish oscillatory solutions of the equation (*). Results of this type are numerous, the principle ones being due to Waltman [5], Kartsatos [1] and the references cited there. Here we shall improve the conclusion of our earlier result ([3], Th. 1) by using the theorem ([1], Th. 3). All functions considered are supposed to be continuous on their domains. In what follows, we consider only such solutions which are nontrivial for all large $t$.

We shall consider the differential equation $(*)$ and the following conditions:

(a) $f$ is a positive function defined on the interval $I=\left[t_{0},+\infty\right)$ with $t_{0} \geqslant 0$ and

$$
\int_{t_{0}}^{+\infty} f(t) d t=+\infty
$$

( $\beta$ ) $g$ is defined on $R^{n}$; sgn $g\left(x_{1}, x_{2}, \ldots, x_{n}\right)=\operatorname{sgn} x_{1}$ for any $\left(x_{1}, x_{2}, \ldots, x_{n}\right) \in R^{n}$; and $g\left(\lambda x_{1}, \lambda x_{2}, \ldots, \lambda x_{n}\right)=\lambda^{2 p+1} g\left(x_{1}, x_{2}, \ldots, x_{n}\right)$ for any $\left(x_{1}, x_{2}, \ldots, x_{n}\right) \in R^{n}$, and $\lambda \in R$ and some nonnegative integer $p$.

Then our improved theorem reads as follows.

Theorem 1. Suppose that the equation ( $*$ ) satisfies $(\alpha)$ and $(\beta)$, where $n$ is odd. Then, any solution of $(*)$ on the interval $I$ is oscillatory, whenever it has a zero. Moreover, every non-oscillatory solution tends to zero monotonically as $t \rightarrow \infty$.

As a first step towards the proof of Theorem 1, we mention the following lemma ([1], Th. 3).

Lemma. Consider (*) with $n$ odd, and moreover,

$$
\begin{aligned}
& f: I \rightarrow R_{+}=(0,+\infty) ; \\
& g: R^{n} \rightarrow R \text { and such that }
\end{aligned}
$$

*De partment of Mathematics, Ibaraki University, Mito, Japan 
Condition $(G)$ :

$x_{1} g\left(x_{1}, x_{2}, \ldots, x_{n}\right)>0$ for every $\left(x_{1}, x_{2}, \ldots, x_{n}\right) \in R^{n}$ with $x_{1} \neq 0$, and for every $\left(x_{1}\right.$, $\left.x_{2}, \ldots, x_{n}\right) \in R^{n}$, and every $\lambda \geqslant K(=$ fixed positive constant $), g\left(-x_{1},-x_{2}, \ldots,-x_{n}\right)$ $=-g\left(x_{1}, x_{2}, \ldots, x_{n}\right)$, and $g\left(\lambda, \lambda x_{2}, \lambda x_{3}, \ldots, \lambda x_{n}\right)=\lambda^{\gamma} g\left(1, x_{2}, \ldots, x_{n}\right)$, where $\gamma=q / r$, $q, r$ odd positive integers relatively prime;

is satisfied.

Then under any one of the following conditions, every solution of $(*)$ is oscillatory or tending monotonically to zero as $t \rightarrow+\infty$ :

b)

$$
\begin{aligned}
& r<1, \int_{t_{0}}^{+\infty} t^{\gamma(n-1)} f(t) d t=+\infty \\
& \gamma=1, \int_{t_{0}}^{+\infty} t^{n-1-\varepsilon} f(t) d t=+\infty, \text { for some } \varepsilon>0 ; \\
& r>1, \int_{t_{0}}^{+\infty} t^{n-1} f(t) d t=+\infty
\end{aligned}
$$

c)

Proof of Theorem 1. The hypotheses of Theorem 1 is the same one as the theorem ([3], Th. 1). Hence, by the conclusion of theorem ([3], Th. 1), we have the result that any solution of $(*)$ on the interval $I$ is oscillatory, whenever it has a zero. Moreover, the hypotheses of Lemma is weaker than the hypotheses of Theorem 1 . Consequently, by the conclusion of Lemma, non-oscillatory solution tends to zero monotonically as $t \rightarrow+\infty$.

2. The purpose of this section is to point out that by using the same argument of the theorem of Lazer [2] is valid for

$$
y^{\prime \prime \prime}-p(x) f\left(y, y^{\prime}, y^{\prime \prime}\right)=0
$$

with some conditions for $p(x)$ and $f\left(y, y^{\prime}, y^{\prime \prime}\right)$.

Theorem 2. If $p(x)$ is continuous and positive (or negative) in the interval $I:[a$, $b]$ and $f\left(y_{1}, y_{2}, y_{3}\right)$ is defined in $R^{3}$ and if for all $y_{1} \in R, y_{1} f\left(y_{1}, y_{1 .}, y_{8}\right)>0$ for $y_{1} \neq 0$, then no solution $y \neq 0$ of (**) in $(a, b)$ can be tangent to $y=0$ more than once.

Proof. Let $y(x) \not \equiv 0$ be a solution of $(* *)$ valid in $(a, b)$ and consider (A)

$$
V(x)=y^{\prime \prime} y-\frac{1}{2}\left(y^{\prime}\right)^{2}
$$

Since $V^{\prime}(x)=y^{\prime \prime \prime}(x) y(x)+y^{\prime \prime} y^{\prime}-y^{\prime} y^{\prime \prime}$

$$
\begin{aligned}
& =y^{\prime \prime \prime} y \\
& \left.=p(x) f\left(y(x), y^{\prime}(x), y^{\prime \prime}(x)\right) y(x)>0 \text { for } y \neq 0, \text { (or } V^{\prime}<0\right) .
\end{aligned}
$$

$V(x)$ is a continuous strictly increasing (or decreasing) function. Hence $V(x)$ has at most one zero. As $y(x)=y^{\prime}(x)=0$ implies that $V(x)=0$, such an $x \in(a, b)$ can not exist more than once. Hence the theorem is completely proved. 
Theorem 3. Under the hypotheses of the previous theorem, no solution of (**) is periodic.

Proof. If $y(x)$ is a periodic solution of (**) with period $t>0$, then

$$
V(x)=y^{\prime \prime}(x) y(x)-\frac{1}{2}\left(y^{\prime}(x)\right)^{2}
$$

is continuous and periodic of period $t$.

As in the proof of Theorem 2,

$$
\left.V^{\prime}(x)>0 \text { (or }<0\right) \text { for } y(x) \neq 0 \text {; }
$$

hence $V(x)$ never decreases (or increases) and can not be periodic. Therefore no solution of (**) is periodic. Thus the theorem is completely proved.

Remark. The Theorem 2 and Theorem 3 are some generalization of the results of Utz [4].

\section{References}

[1] A. G. Kartsatos, Criteria for oscillation of solutions of differential equations of arbitrary order, Proc. Japan Acad., 44 (1968), 599-602.

[2] A. C. Lazer, Problem 5195, Amer. Math. Monthly, 72 (1965), 433.

[3] H. Onose, Oscillatory property of certain non-linear ordinary differential equations. II, Proc. Japan Acad., 44 (1968), 876-878.

[4] W. R. Utz, Nonperiodicity of solutions of a third order equation, Amer. Math. Monthly, 74 (1967), 705-706.

[5] P. Waltman, Oscillation criteria for third order nonlinear differential equations, Pacific J. Math., 18 (1966), 385-389. 